\title{
EFEITO DA TEMPERATURA E PRESSÃO NA EXTRAÇÃO DE ÓLEO DE MILHO EM CONDIÇÕES SUPERCRÍTICAS
}

\author{
A. B. B. L. AIRES ${ }^{1}$, C. M. MARINHO ${ }^{1}$, C. E. HORI ${ }^{1}$, E. O. WATANABE ${ }^{1}$ \\ ${ }^{1}$ Universidade Federal de Uberlândia, Faculdade de Engenharia Química \\ E-mail para contato: erika@ufu.br
}

\begin{abstract}
RESUMO - O milho é considerado o cereal que se transforma no maior número de produtos industrializados, sendo largamente empregado na alimentação humana e animal. No entanto, a extração do óleo de milho é comumente realizada pelo uso de solventes orgânicos, que emitem produtos tóxicos durante o processo de extração, representando assim uma séria ameaça ao meio ambiente. Diante disso, destaca-se a extração do óleo em condições supercríticas, como uma tecnologia inovadora, ambientalmente amigável e que possui maior eficiência de extração. O dióxido de carbono é o solvente mais utilizado, já que possui pressão e temperatura críticas moderadas e não é tóxico. O objetivo deste trabalho foi avaliar o efeito da temperatura e pressão no rendimento do óleo extraído do milho em condições supercríticas. Os ensaios experimentais foram realizados em temperaturas entre 313 e $363 \mathrm{~K}$ mantendo-se a pressão e a vazão constantes em 250 bar e 3,0 $\mathrm{mL} / \mathrm{min}$, respectivamente e também com pressões variando de $150 \mathrm{a}$ 250 bar, vazão de $3,0 \mathrm{~mL} / \mathrm{min}$ e temperatura de $358 \mathrm{~K}$, ambas constantes. Os resultados demonstraram que o aumento da temperatura e pressão de extração levou a um aumento no rendimento do óleo, já que a solubilidade dos compostos no fluido supercrítico é dependente da temperatura e pressão do processo.
\end{abstract}

\section{INTRODUÇÃO}

O milho é considerado o cereal que se transforma no maior número de produtos industrializados, sendo largamente empregado na alimentação humana e animal. Seu óleo é considerado uma excelente fonte de ácidos graxos essenciais e de vitamina E, além de oferecer excelente estabilidade oxidativa.

A extração do óleo de milho é tradicionalmente realizada através de métodos químicos e físicos. Nestes métodos convencionais, o óleo é removido do gérmen do milho moído através de um processo de aquecimento, seguido por prensagem mecânica e pela extração com o uso de solventes orgânicos, por exemplo, hexano, clorofórmio, metanol, dentre outros (Mandarino et al., 2015). Este método convencional é bastante utilizado, porém o uso de solventes, leva preocupação quanto à saúde, à segurança e em relação aos resíduos associados a este processo devido à toxicidade destes solventes (Nobre et al, 2013). Além disso, um aumento crescente no consumo de óleos deve ocorrer devido ao aumento populacional. Consequentemente, a extração convencional do óleo com o uso de solventes orgânicos exigirá maiores quantidades de substâncias tóxicas e prejudiciais ao meio ambiente, como o hexano.

Diante disso, o interesse pela extração com o uso de solventes em condições supercríticas tem sido crescente nos últimos anos devido às limitações legais relacionadas às quantidades 
de resíduos de solventes orgânicos que podem ser descartadas ao ambiente e às quantidades que podem estar contidas em alimentos e fármacos. Por outro lado, os solventes supercríticos podem ser utilizados nas indústrias alimentícias, de bebidas e farmacêuticas, o que torna esta técnica bastante atraente. Além disso, nenhum processo adicional é necessário para a remoção do solvente supercrítico (geralmente, o dióxido de carbono) do extrato ou dos resíduos sólidos e líquidos (Marr e Gamse, 2000).

Um fluido é definido como supercrítico quando apresenta a temperatura e pressão acima de seu ponto crítico, em que existe apenas uma única fase e com propriedades intermediárias de gases e líquidos. O dióxido de carbono supercrítico $\left(\mathrm{CO}_{2}\right)$ é o solvente mais utilizado neste tipo de extração, pois apresenta as seguintes vantagens: sua moderada pressão crítica (72,9 bar) permite um custo menor para a compressão do fluido, sua baixa temperatura crítica $\left(31,1^{\circ} \mathrm{C}\right)$ permite a extração de frações de lipídeos que são termicamente sensíveis, sem que ocorra degradação e sua baixa toxicidade, baixa flamabilidade e reatividade desprezível facilita a extração do óleo em segurança (Santana et al., 2012).

A principal desvantagem do método de extração em condições supercríticas, comparando-se às demais técnicas existentes, é o alto custo de investimento da infraestrutura e operacional em relação às técnicas de extração tradicionais a pressão atmosférica. Para que este processo de extração em condições supercríticas seja uma alternativa viável para extração do óleo é necessário um estudo mais amplo deste tipo de extração e das variáveis envolvidas no processo, sendo as principais: temperatura, pressão, tempo de extração e vazão de alimentação de $\mathrm{CO}_{2}$. Estas variáveis necessitam ser otimizadas para um processo de extração eficiente (Friedrich e Pryde, 1984).

Em geral, o rendimento da extração aumenta com a pressão devido ao aumento da solubilidade do óleo no $\mathrm{CO}_{2}$ ocasionado pelo aumento da densidade do dióxido de carbono em condições supercríticas. A temperatura também afeta a extração supercrítica, sendo que seu impacto sobre a solubilidade é complexo e dependente de parâmetros competitivos densidade do $\mathrm{CO}_{2}$ e pressão de vapor do soluto. Enquanto que a densidade de $\mathrm{CO}_{2}$ diminui com a temperatura, a pressão de vapor dos solutos aumenta, levando ao fenômeno bastante conhecido de "cross-over" para isotermas de solubilidade. Portanto, o impacto resultante da temperatura na solubilidade é definido pelo parâmetro mais significativo (densidade do $\mathrm{CO}_{2}$ ou pressão de vapor) uma dada pressão (Temelli, 2009). Além disso, a difusividade aumenta com a temperatura, o que leva ao aumento da cinética de transferência de massa durante a extração.

O presente trabalho visou o estudo da extração do óleo de milho utilizando-se o dióxido de carbono em condições supercríticas através da avaliação dos parâmetros temperatura, pressão e tempo para a completa extração do óleo.

\section{MATERIAL E MÉTODO}

\subsection{Material}

Para a extração supercrítica do óleo foi utilizado gérmen de milho previamente moído cedido pela Caramuru Alimentos S.A. e dióxido de carbono de pureza 99,8\% (Gama Gases Especiais - IBG 99\%). 


\subsection{Método}

Extração com o uso de $\mathrm{CO}_{2}$ em condições supercríticas:As extrações foram conduzidas na unidade experimental descrita na Figura 1, composta de: um cilindro de $\mathrm{CO}_{2}$, bomba seringa (modelo Isco), que alcança pressões de até 258 bar e vazão de $200 \mathrm{~mL} / \mathrm{min}$, dois banhos termostáticos e um extrator encamisado. Três válvulas tipo agulha foram adicionadas para separar cada parte do equipamento e uma válvula micrométrica foi utilizada para regular a vazão de $\mathrm{CO}_{2}$. Um controlador de temperatura foi usado para aquecer a válvula micrométrica e evitar o congelamento devido à expansão do $\mathrm{CO}_{2}$.

Figura 1 - Esquema da unidade experimental a ser utilizada para a extração com fluido supercrítico

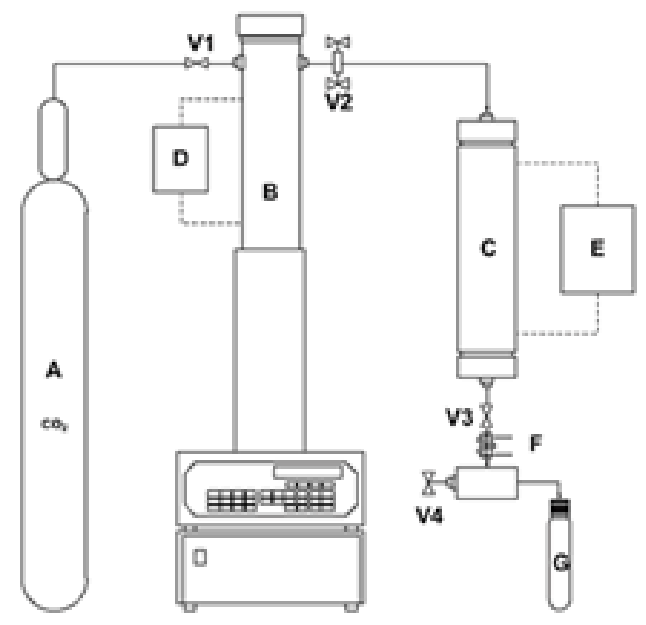

(A) cilindro de gás;

(B) bomba de alta pressão;

(C) extrator;

(D, E) banho termostatizado;

(V1, V2 e V3) válvulas agulhas;

(V4) válvula micrométrica;

(F) controlador de temperatura da válvula de expansão;

(G) frasco de coleta das amostras

O gérmen de milho foi inserido em aproximadamente $25 \mathrm{~g}$ no extrator. A bomba de alta pressão foi resfriada para $273 \mathrm{~K}$ e então preenchida com $\mathrm{CO}_{2}$. $\mathrm{O}$ gás foi preenchido no extrator durante 30 minutos para evitar gradientes de temperatura e pressão. $\mathrm{O}$ extrator e a válvula de expansão foram aquecidos nas temperaturas desejadas. A válvula V1 foi fechada, a válvula V2 aberta e o gás foi pressurizado a 250 bar durante 30 minutos antes do início da extração. A massa do frasco vazio foi medida a cada 10 minutos para a realização da cinética da extração. O rendimento foi calculado como: massa de extrato dividida pela massa inicial. Os experimentos foram conduzidos até que a massa de extrato permanecesse constante. Os ensaios foram realizados em duplicata.

\section{RESULTADOS E DISCUSSÕES}

\subsection{Efeito da temperatura}

A influência da temperatura no rendimento e no tempo de extração do óleo de milho em condições supercríticas é apresentada na Figura 2. A pressão e a vazão foram mantidas constantes a 250 bar e $3 \mathrm{~mL} / \mathrm{min}$, respectivamente. Na Tabela 1, verifica-se os valores de rendimento total e de solubilidade do óleo a diferentes valores de temperatura $(313-363 \mathrm{~K})$ e a Figura 2 mostra o rendimento do óleo de milho extraído com o passar do tempo a diferentes valores de temperatura. 
Figura 2 - Efeito da temperatura sobre o rendimento da extração de óleo de milho a pressão de 250 bar e vazão de $\mathrm{CO}_{2}$ de $3 \mathrm{~mL} / \mathrm{min}$

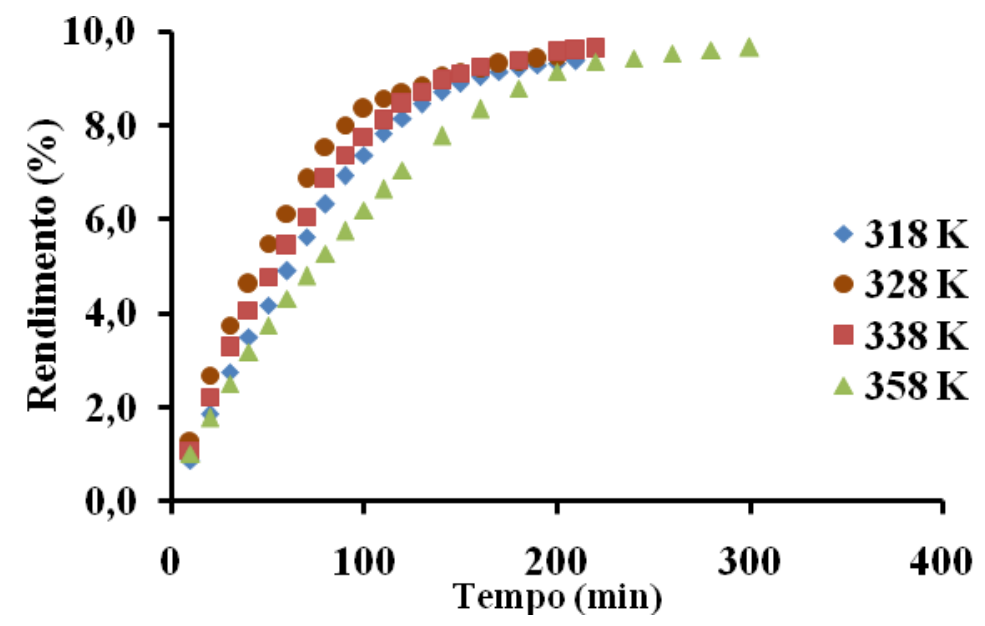

Tabela 1 - Rendimento total e solubilidade obtidos nas extrações do óleo de milho com $\mathrm{CO}_{2}$ em condições supercríticas, a pressão de 250 bar e vazão de $\mathrm{CO}_{2}$ de $3 \mathrm{~mL} / \mathrm{min}$.

\begin{tabular}{|c|c|c|c|}
\hline Ensaio & Temperatura $(\mathrm{K})$ & Rendimento $(\%)$ & Solubilidade $\left(\mathrm{g}\right.$ de extrato/g de $\left.\mathrm{CO}_{2}\right)$ \\
\hline \hline 1 & 318 & 9,38 & 0,0918 \\
\hline 2 & 328 & 9,47 & 0,0631 \\
\hline 3 & 338 & 9,65 & 0,0490 \\
\hline 4 & 358 & 9,67 & 0,0448 \\
\hline
\end{tabular}

Verificou-se que os rendimentos da extração foram aproximadamente iguais com a variação da temperatura, alcançando-se um rendimento máximo de aproximadamente $9 \%$. No entanto, o tempo de extração foi distinto para as diferentes temperaturas utilizadas. Para as temperaturas de 318 a $338 \mathrm{~K}$, o tempo de extração foi de aproximadamente $200 \mathrm{~min}$, enquanto que para a temperatura de $358 \mathrm{~K}$ foi necessário um tempo de 300 min para alcançar o mesmo rendimento de cerca de $9 \%$. Estes resultados podem ser explicados pela redução da solubilidade do óleo no solvente $\mathrm{CO}_{2}$ para a temperatura de $358 \mathrm{~K}$ o que levou ao aumento do tempo de extração a fim de atingir o rendimento máximo de óleo.

A solubilidade de um composto pode ser explicada por dois mecanismos concorrentes: o aumento da temperatura aumenta a pressão de vapor do soluto e aumenta a solubilidade do soluto no solvente. Porém, o aumento da temperatura também ocasiona a diminuição da densidade do solvente e a redução da solubilidade do soluto no solvente. Na pressão de 250 bar utilizada, observou-se que a solubilidade diminuiu com o aumento da temperatura, ou seja, o efeito da diminuição da densidade do solvente sobrepõe-se ao efeito do aumento da pressão de vapor do soluto. Estes resultados encontram-se de acordo com o cross-over já relatado por Friedrich e Pryde. (1984) e Brunner (2005).

\subsection{Efeito da pressão}

Ensaios experimentais foram realizados para se observar o efeito da pressão $(150-250$ bar) no rendimento do óleo de milho e no tempo de extração (Figura 3). A vazão e a 
temperatura foram mantidas constantes a $3 \mathrm{~mL} / \mathrm{min}$ e $358 \mathrm{~K}$, respectivamente. Na Tabela 2, tem-se os valores de rendimento total e de solubilidade do óleo a diferentes valores de pressão (150 - 250 bar).

Figura 3 - Efeito da temperatura sobre o rendimento da extração de óleo de milho a temperatura de $358 \mathrm{~K}$ e vazão de $\mathrm{CO}_{2}$ de $3 \mathrm{~mL} / \mathrm{min}$

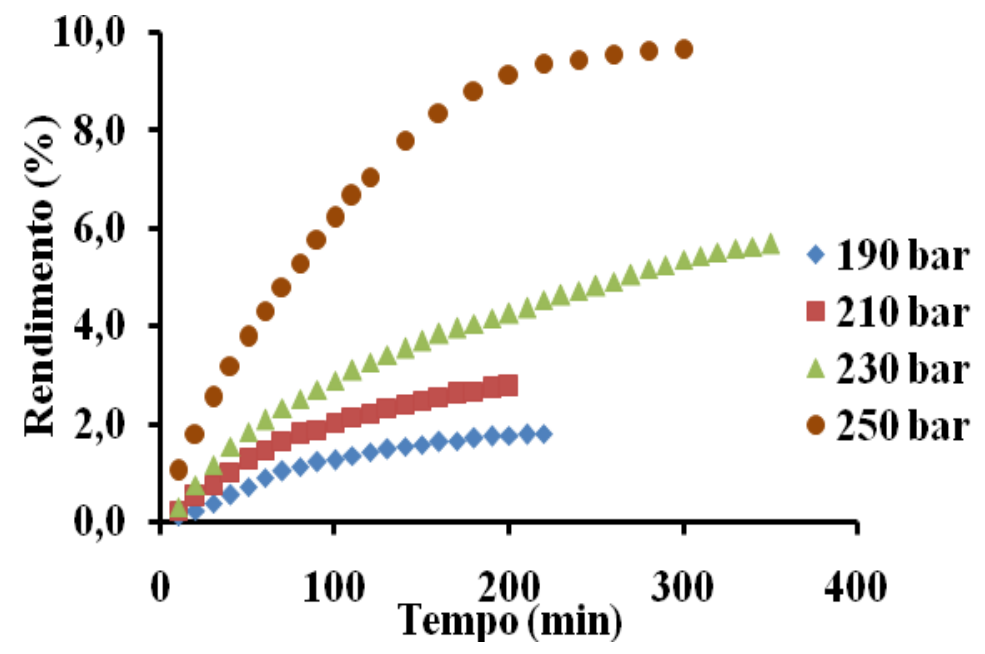

Tabela 2 - Rendimento total e solubilidade obtidos nas extrações do óleo de milho com $\mathrm{CO}_{2}$ em condições supercríticas, a temperatura de $358 \mathrm{~K}$ e vazão de $\mathrm{CO}_{2}$ de $3 \mathrm{~mL} / \mathrm{min}$.

\begin{tabular}{|c|c|c|c|}
\hline Ensaio & Pressão (bar) & Rendimento $(\%)$ & Solubilidade (g de extrato/g de $\left.\mathrm{CO}_{2}\right)$ \\
\hline \hline 1 & 190 & 1,79 & 0,0197 \\
\hline 2 & 210 & 2,75 & 0,0347 \\
\hline 3 & 230 & 5,67 & 0,0492 \\
\hline 4 & 250 & 9,67 & 0,0448 \\
\hline
\end{tabular}

Verificou-se que, à temperatura constante de $358 \mathrm{~K}$, quanto maior a pressão, maior o rendimento obtido na extração. Estes resultados estão em concordância com Rebolleda et al. (2012), em que o rendimento da extração aumenta com a pressão devido ao aumento da solubilidade do óleo no $\mathrm{CO}_{2}$ ocasionada pelo aumento da densidade do dióxido de carbono em condições supercríticas. Observou-se também que para pressões de 230 e 250 bar foi necessário um tempo de extração de 300 min para alcançar o máximo rendimento do processo.

\section{CONCLUSÃO}

Neste trabalho, o efeito dos parâmetros temperatura e pressão foi avaliado na extração do óleo de milho em condições supercríticas. A variação da temperatura mostrou não ter influência significativa sobre o rendimento do óleo extraído, mas leva à redução da solubilidade do óleo no solvente $\mathrm{CO}_{2}$, a uma pressão constante de 250 bar e vazão de 3 $\mathrm{mL} / \mathrm{min}$. Os ensaios para verificação do efeito da pressão sobre o rendimento da extração evidenciaram que o rendimento da extração aumenta com a pressão devido ao aumento da solubilidade do óleo no $\mathrm{CO}_{2}$ em condições supercríticas. 


\section{REFERÊNCIAS BIBLIOGRÁFICAS}

BRUNNER, G. Supercritical fluids: technology and application to food processing. J. Food Eng., v. 67, p. 21-33, 2005.

FRIEDRICH, J. P.; PRYDE, E. H. Supercritical $\mathrm{CO}_{2}$ extraction of lipid-bearing materials and characterization of the products. J. Am. Oil Chem. Soc., v. 61, p. 223-228, 1984.

MANDARINO, J.;MARCOS, G.; ROESSING, A. C. Tecnologia para produção do óleo de soja: descrição das etapas, equipamentos, produtos e subprodutos. Londrina : Embrapa Soja, 2001. p. 40.

MARR, R., GAMSE, T. Use of supercritical fluids for different processes including new developments - a review. Chem. Eng. Process., v. 39, p. 19-28, 2000.

NOBRE, B.P.; VILLALOBOS, F.; BARRAGÁN, B.E.; OLIVEIRA, A.C.; BATISTA, A.P.; MARQUES, P.A.S.S.; MENDES, R.L.; SOVOVÁ, H.; PALAVRA, A.F.; GOUVEIA, L. A biorefinery from Nannochloropsis sp. microalga - Extraction of oils and pigments. Production of biohydrogen from the leftover biomass. Bioresour. Technol., v. 135, p. 128-136, 2013.

REBOLlEDA, S.; RUBIO, N.; BELTRÁN, S.; SANZ, M. T.; GONZÁLEZ-SAN JOSÉ, M. L. Supercritical fluid extraction of corn germ oil: Study of the influence of process parameters on the extraction yield and oil quality. J. Supercr. Fluids, v. 72, p. 270-277, 2012.

SANTANA, A.; JESUS, S.; LARRAYOZ, M. A.; FILHO, R. M. Supercritical carbon dioxide extraction of algal lipids for the biodiesel production. Procedia Eng., v. 42, p. 1755 $1761,2012$.

TEMELLI, F. Perspectives on supercritical fluid processing of fats and oils. J. Supercrit. Fluids, v. 47, p. 583-590, 2009.

\section{AGRADECIMENTOS}

Os autores agradecem o auxílio financeiro da FAPEMIG, CNPq, CAPES, VALE S.A. e CARAMURU Alimentos por ceder gentilmente o gérmen de milho utilizado neste trabalho. 